\title{
Binocular depth discrimination depends on orientation
}

\author{
RANDOLPH BLAKE, JOHN M. CAMISA, and DIANNE N. ANTOINETTI \\ Cresap Neuroscience Laboratory, Northwestern University, Evanston, Illinois 60201
}

\begin{abstract}
Using both the method of adjustment and forced-choice techniques, it was found that binocular depth thresholds depend on the orientation of the test targets, which in these experiments consisted of long, thin rods. Stereoacuity is greatest with vertical rods and decreases progressively as the angle of orientation approaches horizontal. The relationship between stereoacuity and orientation is governed by the orientation of the images of the targets on the retina, and is predicted fairiy well by the sine of the angle of orientation, suggesting an equivalence between angular rotation and reductions in rod length.
\end{abstract}

Of the many potential cues for the perception of depth, none surpasses binocular disparity when tiny differences in the distances of objects from the eyes must be discriminated. This very keen sense of stereoscopic depth perception is produced by the dissimilarities between the images on the two retinae, which arise by virtue of the horizontal separation of the eyes in the head. The ability of humans to utilize binocular disparity information has been studied psychophysically under a variety of stimulus conditions (e.g., see Ogle, 1962), and with recent advances in the technology of single-cell recording, we have begun to see how the visual nervous system encodes information necessary for stereopsis (Barlow, Blakemore, \& Pettigrew, 1967; Hubel \& Wiesel, 1970; Nikara, Bishop, \& Pettigrew, 1968).

One aspect of stereopsis which has received little attention is the influence of orientation on binocular depth discrimination. In the psychophysics laboratory, it is customary to measure stereoscopic thresholds using vertically oriented test targets, an altogether reasonable choice since horizontal disparity, the fundamental cue for stereopsis, can result from vertical, but not horizontal, contours. Yet, as we look about the visual world, it is obvious that our comprehension of stereoscopic depth covers nearly the entire range of orientations. Of course, this observation seems quite reasonable when we consider the geometry of the situation. To illustrate, imagine a Cartesian coordinate system superimposed upon objects in your visual field. Among the various objects, contours of all orientations except horizontal will produce nonzero vertical projections onto the imaginary ordinate. In principle, that proportion of

This work was supported by NIH Grants EY-00321 and S05-RR07028. We are grateful to R. Reed and E. Levinson for serving as observers and to Robest Sekuler for helpful discussion. Requests for reprints should be sent to Randolph Blake, Department of Psychology, Cresap Neuroscience Laboratory, Northwestern University, Evanston, Illinois 60201. a contour defined by this vertical projection could give rise to horizontal disparities between the leftand right-eye images of contours at different distances, thus producing the perception of stereoscopic depth.

Carrying this geometrical analysis one step further, it also is true that, for any particular contour, the ordinate value for the vertical projection will become progressively smaller with angular rotations of that contour from vertical to horizontal. So, in effect, the proportion of the contour which can contribute to a horizontal disparity would be reduced, by an amount given by the absolute value of the sine of the angle of orientation. This point is illustrated in Figure 1. Now it has been shown that binocular depth thresholds increase with actual reductions in the length of vertical lines (e.g., Andersen \& Weymouth, 1923), so one might expect binocular depth thresholds for lines of fixed length to vary also with angular rotation, because of changes in
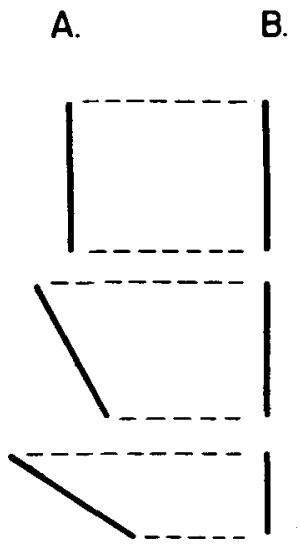

Figure 1. The lines in column $A$ are meant to illustrate a contour in the visual world seen at three different orientations. Column B shows the projection of this contour onto the ordinate of an imaginary Cartesian coordinate system. As the contour is rotated from vertical toward horizontal (A), this vertical projection (B) becomes shorter and shorter. 
the effective proportion of the contour which could contribute to horizontal disparity. By measuring stereoscopic acuity over a range of orientations, we have found this to be true. Those results, along with additional data concerning the relationship between stereoacuity and orientation, are presented in this paper.

\section{METHODS}

\section{Apparatus}

In these experiments, a modified three-rod test was used to measure binocular depth thresholds. The apparatus, which is illustrated in Figure 2, consisted of two light-tight viewing tubes, one which contained three slender, vertical rods seen silhouetted against a uniformly transilluminated white background $\left(20 \mathrm{~cd} / \mathrm{m}^{2}\right)$ and the other which contained an identical background display $\left(20 \mathrm{~cd} / \mathrm{m}^{2}\right)$ but without rods. These two displays were optically superimposed via a beam splitter and could be presented independently to either or both eyes by appropriate arrangement of polarizing filters situated immediately in front of the eyes. With this arrangement, it was possible to measure monocular and binocular depth thresholds without affecting pupil diameter, since the state of light adaptation of the eyes was constant.

Figure 3 illustrates the subject's view of the display. The two flanking rods were securely attached to one viewing tube, but the center rod was mounted on a rack and pinion device located just under this tube, out of view of the subject. By activation of a bidirectional, slow-synchronous motor which operated the rack and pinion, the center rod could be moved directly away from or toward the eyes at the rate of $2 \mathrm{~mm} / \mathrm{sec}$. With all three rods arrayed in the fronto-parallel plane, the viewing distance was exactly $300 \mathrm{~cm}$, which produced the angular subtenses given in Figure 3. The subject viewed the display through small,

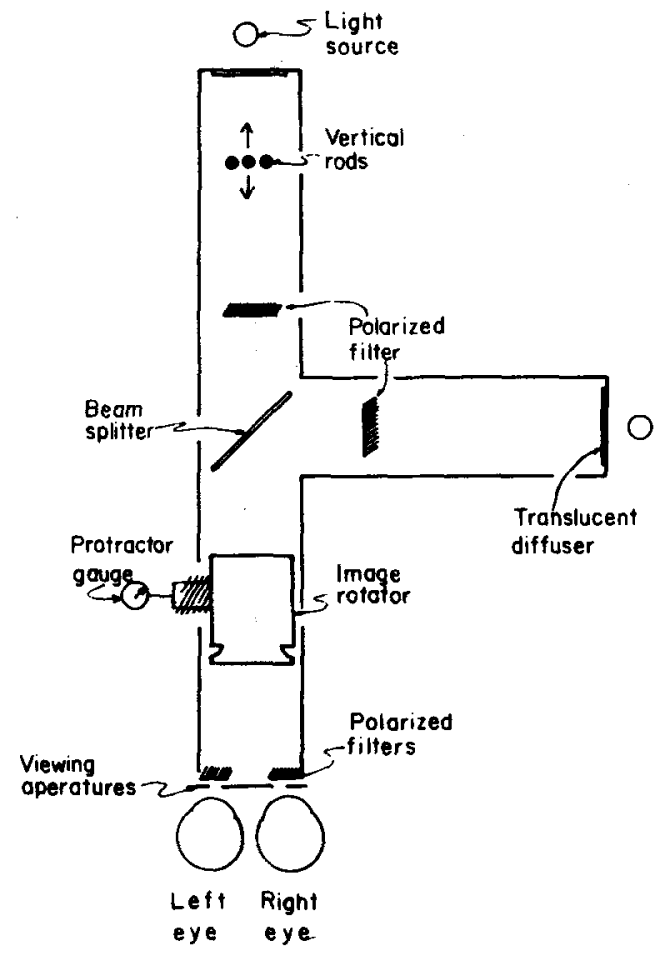

Figure 2. Schematic of the apparatus, the details of which are given in the text.

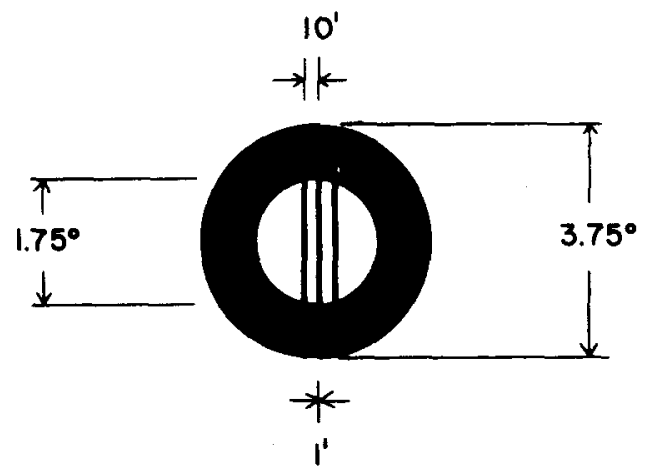

Figure 3. Diagram, not to scale, of the subject's view of the three-rod display. The distance from the eyes to the rods, with all three in the same fronto-parallel plane, was $300 \mathrm{~cm}$, which produced the visual angles shown. The background portion of the display, against which the rods were seen, was located $312 \mathrm{~cm}$ from the eyes and subtended the visual angles shown. The circular aperture contained a plate of white Plexiglas which was transilluminated by an incandescent bulb, which provided the only source of illumination with in the viewing tunnel. Neither end of the rods was visible to the subject, nor were there any shadows.

circular apertures while firmly positioned on a dental impression board.

Located in the viewing path was a large dove-type prism (face dimensions: $66 \times 79 \mathrm{~mm}$ ) which, when rotated on longitudinal axis, rotated the entire image of the display. This prism was rigidly mounted in a metal housing and could be turned using a gear mechanism linked to a protractor gauge which displayed angular rotation to within $1^{\circ}$ accuracy.

\section{Psychophysical Procedure}

Two different methods were employed to measure depth thresholds as a function of orientation. In Experiments 1 and 3 , the subject operated a manual switch connected to the bidirectional motor to adjust the position of the center rod until it appeared to lie in the same fronto-parallel plane as the flanking rods. The deviation of this setting from the true fronto-parallel plane at $300 \mathrm{~cm}$ was noted by the experimenter, who then offset the center rod in depth by an unspecified amount. Ten adjustments were made for each orientation, and the threshold was defined as the standard deviation of these settings, converted into the equivalent disparity angle (Ogle, 1962).

In Experiment 2, a double-random staircase method (Cornsweet, 1962) was used. On each trial, the center rod was fixed in depth relative to the flanking rods and the subject reported whether this center rod appeared "in front of" or "behind" the other two. Following correct responses, the distance between the center and flanking rods was reduced by a fixed amount; following incorrect responses, this distance was increased by the same amount. Two series of trials were run concurrently, one which began with the center rod clearly in the near position and the other beginning with the center rod clearly in the far position. Throughout a sequence of trials, the experimenter randomly switched from one staircase (e.g., the near-depth series) to the other (the far-depth series), so that the subject was unable to anticipate the depth settings from trial to trial. On each trial, the subject was allowed free eye movements and unlimited viewing time, in order to encourage maximal utilization of disparity information; usually judgments were made within $5 \mathrm{sec}$. Between trials, while the experimenter positioned the center rod for the next trial, the subject viewed the blank field. The two staircase series were continued until $\mathbf{4 0}$ judgments were obtained at depth values producing equivocal responses. Near-depth and far-depth 
thresholds were then estimated from frequency-of-seeing curves plotted in the manner described by Ogle (1962).

\section{Subjects}

A total of four people, including the authors, participated in one or more of the experiments. All had normal or corrected-tonormal vision $(20 / 20)$ and no problems with binocular fusion or stereopsis. The results in each experiment were qualitatively similar for all subjects, and the results presented in Figures 4-7 are quite typical.

\section{Sign Convention for Specifying Orientation}

In our figures, $90^{\circ}$ refers to the condition where the rods were seen vertically oriented, as in Figure 3 . Angular rotations in the counterclockwise direction are represented by angles greater than $90^{\circ}$. Thus $135^{\circ}$ refers to diagonal rods; $180^{\circ}$ is the horizontal orientation; $270^{\circ}$ is vertical and, from the subject's viewpoint, indistinguishable from $90^{\circ}$. Following Experiment 1, in which we confirm that the relationship between stereo-threshold and orientation is approximately symmetrical about $180^{\circ}$, we confined our measurements to orientations between $90^{\circ}$ and $180^{\circ}$.

\section{Threshold Metric}

Thresholds for stereoscopic depth are conventionally defined in terms of the angular disparity produced by objects which are just noticeably displaced in sagittal distance. For depth tests utilizing real objects in visual space, not some type of haploscopic device, angular disparity is given by the convergence angle for the nearer object minus that for the farther object, a value which is derived simply from geometry (e.g., see Graham, 1965). It is this measure of threshold, angular disparity expressed in seconds of arc, that we have used in this paper.

\section{RESULTS AND DISCUSSION}

\section{Experiment 1}

In this experiment, the method of adjustment was used to measure depth thresholds for orientations ranging from $90^{\circ}$ to $270^{\circ}$, in $10^{\circ}$ increments, and the typical pattern of results is shown in Figure 4. First, notice the three values plotted at $90^{\circ}$ : the open

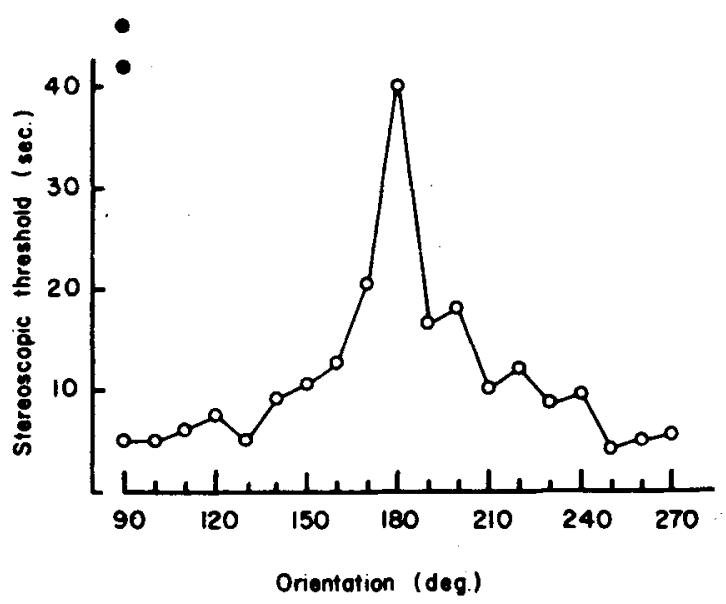

Figure 4. Stereoscopic thresholds at different orientations. Each point represents the standard deviation of the subject's equal distance settings $(n=10)$, expressed in terms of seconds of disparity. The half-filled symbols at $90^{\circ}$ (vertical rods) show the depth thresholds measured monocularly $(0$ - left eye, 0 right eye). Subject E.L. symbol represents the binocular threshold and the half-filled symbols indicate the thresholds measured with monocular viewing, which, of course, eliminates binocular disparity as a source of depth information. Here it can be seen that there is approximately a tenfold difference between the minimum depth discriminated on the basis of monocular cues (e.g., image size) and that discriminated binocularly. Among the three observers who participated in this experiment, binocular thresholds at $90^{\circ}$ varied from 3.75 to $7.4 \mathrm{sec}$ of arc. These values are in good agreement with previous results obtained under comparable conditions (Graham, 1965).

The open symbols in Figure 4 show that binocular depth discrimination is very evidently related to the orientation of the test rods, with thresholds increasing as orientation progresses from vertical to horizontal. The threshold at $180^{\circ}$ is very similar to the values obtained monocularly, indicating that binocular discrimination of depth differences with horizontal rods is based exclusively on monocular cues.

Consider again the geometrical analysis presented earlier. There it was pointed out that contours of any orientation except horizontal can give rise to the sensation of stereoscopic depth, by generating horizontal disparities on the two retinae. Now for a line of fixed length, $\ell$, the proportionate length of the line which can contribute to horizontal disparity is given by the absolute value of $\ell \sin \theta$, where $\theta$ is the orientation of the line. If we assume that increases in binocular depth threshold with angular rotations from vertical to horizontal result from reductions in this proportionate length, then we might expect binocular depth sensitivity to fall off according to a simple sine function:

$$
\frac{1}{\eta_{\theta}}=\frac{1}{v_{\theta}} \cdot \sin \theta
$$

where $\eta_{\theta}$ is the binocular threshold for targets of orientation $\theta$, and $\eta_{v}$ is the binocular threshold for vertically oriented targets. ${ }^{1}$

The extent to which this equation describes our results can be seen in Figure 5. Here we have replotted the threshold points from: Figure 4 as reciprocals on a logarithmic scale, which is conventional for plotting stereoacuity, and have drawn Function 1 as smooth curves symmetrically positioned about $180^{\circ}$. The data points match fairly closely the theoretical function, and in the case of the other two observers who participated in this experiment the fit between obtained and predicted stereoacuities is equally good. These results suggest that essentially all horizontal disparity information, regardless of the orientations of the contours producing those disparities, is utilized in performing relative depth discrimination.

\section{Experiment 2}

During the first experiment observers frequently 


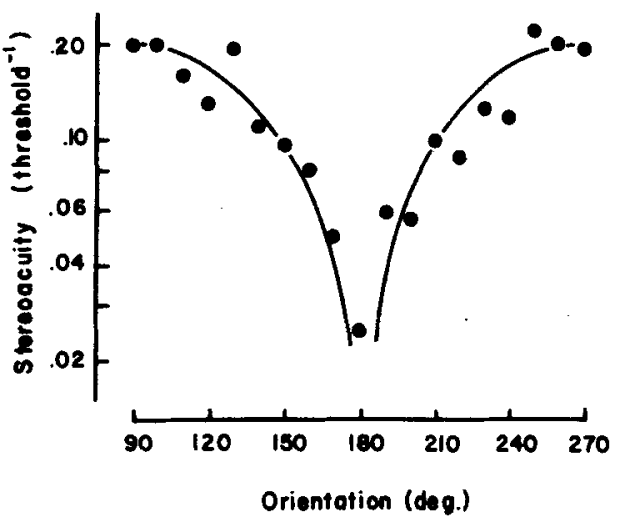

Figure 5. The results in the previous figure have been replotted here as stereoacuity (logarithmic scale), which is the reciprocal of threshold. The continuous curves show the function $\eta_{\theta}^{-1}=\eta_{\theta}^{-1} \sin \theta$.

expressed lack of confidence in the accuracy of their depth settings for orientations near horizontal. It was noted that, at these orientations, there was a relatively large range of actual depths over which the center rod appeared in the same frontal plane as the flanking rods. A common strategy, therefore, was to move the center rod until depth was perceived and then to move the rod in the opposite direction until the sensation of depth first disappeared. This raises the possibility that the threshold estimates, which were based on the variations of these settings, might be influenced by criterion effects. Because we were interested in the particular form of the relationship between stereoacuity and orientation, we decided to repeat the experiment using a forcedchoice technique which, although more timeconsuming than the method of adjustment, is essentially independent of criterion effects.

In this second experiment, depth thresholds were measured for orientations ranging from $90^{\circ}$ to $180^{\circ}$, in $15^{\circ}$ increments, using the double-staircase procedure described in the methods. Threshold values were derived by plotting the proportion of trials on which the center rod was judged nearer than the flanking rods; a smooth curve was fitted by eye to the points, and the stereo-thresholds for near and far depth were defined as the depth value producing a "near" response on $75 \%$ and $25 \%$ of the trials, respectively. In cases where the $50 \%$ level (equal subjective depth) was different from zero relative depth, thresholds were defined as difference scores from the depth value yielding chance performance (i.e., the $50 \%$ level). These departures of equal subjective depth from the $50 \%$ level were small but systematic and probably reflect deviations of the horopter from the objective fronto-parallel plane (Ogle, 1962).

Three subjects participated in this experiment and characteristic results are shown in Figure 6A, open circles for near depth and closed circles for far depth. These threshold estimates are of the same order of magnitude as those obtained with the method of adjustment in Experiment 1. Clearly, there is no systematic difference between near and far thresholds as a function of orientation.

In Figure 6B, we have plotted at each orientation the reciprocal of the average of the near-depth and far-depth thresholds from Figure 6A, and have drawn the predicted fall off in stereoacuity based on the sine function of Equation 1. Again we find a rather good agreement between the predicted and obtained values. The results from these first two experiments suggest that essentially all horizontal disparity information, regardless of the orientations of the contours producing those disparities, is utilized in performing relative depth discriminations.

\section{Experiment 3}

So far, we have shown that binocular depth discrimination very definitely is influenced by contour orientation, in a manner which is predictable on the basis of the geometry of the situation. In describing this relationship between stereoacuity and orientation, however, we have not distinguished between perceived orientation, which usually coincides with the true orientation of objects in the environment defined in terms of gravitational coordinates, and retinal orientation, which refers to the orientation of the image on the retina. Yet this distinction is certainly an important one, for

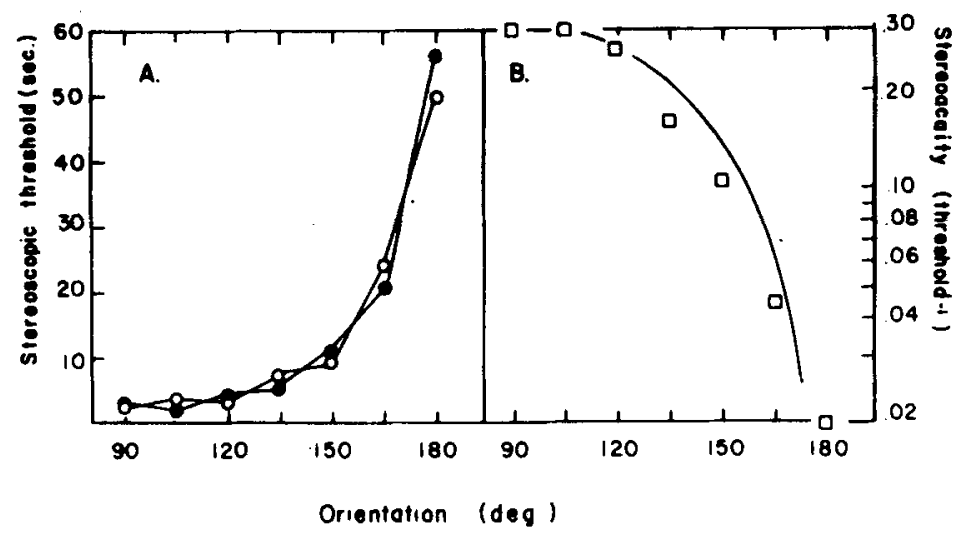

Figure 6. (A) Stereoscopic thresholds for near depth $(O)$ and far depth $(\bullet)$ as a function of the orientation of the lest rods. Fach pair of points at the various orientations was derived from psychometric curve in the manner described in the test. Subject J.C. (b) The means of the near-depth and fardepth thresholds from Figure $6 A$ and replotted as stereoacuity on a logarithmic scale. The smooth curve shows the fall off in stereoacuity predicted by Equation 1. 
it underlies the familiar problem of orientational constancy: the visual world appears to remain upright and stable when the head or body is tilted, even though the orientation of the image on the retina rotates with these movements. This phenomenon of orientational constancy implies the existence of some compensatory mechanism which utilizes proprioceptive information to somehow recalibrate the orientation signified by the retinal image. Now, in view of the strong relationship between binocular depth discrimination and orientation, it should be possible to decide whether this mechanism responsible for orientational constancy occurs before or after the site of stereopsis within the central nervous system, by determining whether stereoacuity varies with the objectivity orientation of targets in visual space, or with the orientation of the images of those targets upon the retina.

To accomplish this, we mounted the biteboard and viewing apertures on a rigid clamp which could be rotated through a $90^{\circ}$ angle. Using the method of adjustment, binocular depth thresholds were measured for three conditions of viewing: (a) with the head rigidly positioned exactly upright $\left(90^{\circ}\right)$, the angle of the rods was varied from $90^{\circ}$ to $180^{\circ}$ in $15^{\circ}$ increments; (b) with the orientation of the rods constant at $90^{\circ}$, the angle of the head was rotated clockwise from vertical $\left(90^{\circ}\right)$ to horizontal $\left(0^{\circ}\right)$ in $15^{\circ}$ increments; and (c) the head and rods were rotated counterclockwise together from vertical to horizontal in $15^{\circ}$ increments. Ten settings were made at each orientation for all conditions. We should point out that tilting the head, as we have done in Conditions $b$ and $c$, introduces the possibility of counterrotational eye movements (e.g., Miller, 1962) which would alter the orientation of the retinal image a little. However, in plotting the results, we have elected to ignore this factor since the possible error involves only a few degrees of angular rotation (Mitchell \& Blakemore, 1972).

Results from this experiment are given in Figure 7. Here it can be seen that changes in the orientation of the images on the retina influence binocular depth thresholds, regardless whether these changes result from rotation of the test rods in visual space (open symbols) or from tilting of the head (filled symbols). When rotation of the rods in visual space is accompanied by an equivalent degree of head rotation, thus maintaining the images of the rods vertical on the retinae, depth thresholds are unchanged (halffilled symbols). This pattern of results indicates, then, that stereopsis is based exclusively on retinal image informtion which is uncorrected for head and body tilt. The mechanism responsible for orientation constancy therefore must occur at some subsequent stage in the visual pathways. This conclusion is consistent with results from pattern adaptation experiments, the results of which demonstrate that

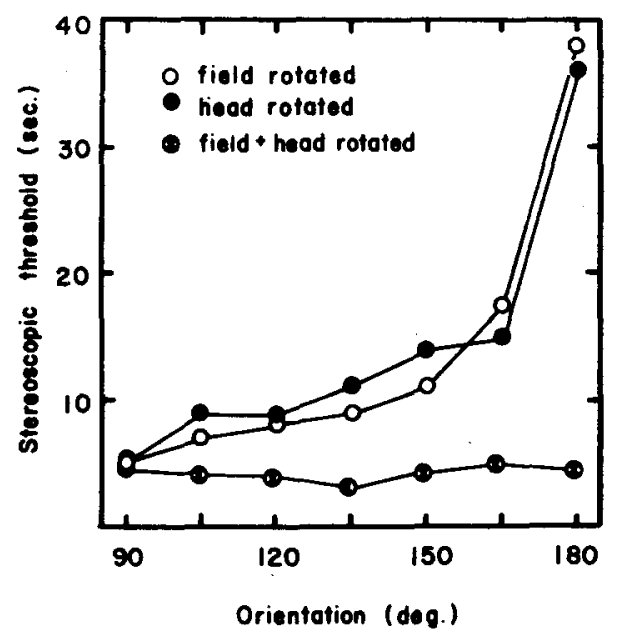

Figure 7. The relationship between stereoscopic threshold and orientation for three viewing conditions. The thresholds shown with open circles were measured with the head upright and the display rotated to the various orientations, just as in Experiments 1 and 2; completely filied symbols show the results when the display remained vertical but the head was rotated; and the half-filled symbols are thresholds with both the head and the display rotated to the same angle. Each point is based on the standard deviation of 10 equal distance settings. Subject R.B.

the site of disparity-specific visual aftereffects (Blakemore \& Hague, 1972; Mitchell \& Baker, 1973) precedes the mechanism responsible for visual orientation constancy (Findlay \& Parker, 1972; Mitchell \& Blakemore, 1972).

\section{CONCLUSION}

The accuracy of stereoscopic depth discrimination depends on a number of factors, including retinal illumination (Mueller \& Lloyd, 1948), lateral separation between test lines (e.g., Graham, Riggs, Mueller, \& Solomon, 1949), similarity in shape of the test lines (Mitchell \& O'Hagan, 1972), and the length of the test lines (e.g., Andersen \& Weymouth, 1923). To this list of influencing factors we now can add the orientation of the retinal images of the test lines. The results in Figures 5 and $6 \mathrm{~B}$ suggest that the relationship between stereoacuity and orientation can be described by a simple sine function. One way to look at this relationship, then, is to consider a nonvertical line as equivalent to a vertically oriented line of reduced length, with the proportion of reduction given by sine $\theta$. This idea of an equivalence between line orientation and line length, in terms of stereoacuity, is one we are currently-studying in greater detail.

\section{REFERENCES}

Andersen, E. E., \& Wermouth, F. W. Visual perception and the retinal mosaic. I. Retinal mean local sign - an explanation of the fineness of binocular perception of distance. American Journal of Physiology, 1923, 64, 561-594. 
Barlow. H. B., Blakemore, C., \& Pettigrew, J. D. The neural mechanism of binocular depth discrimination. Journal of Physiology. 1967. 193, 327-342.

Blakemore, C., \& HAGUE, B. Evidence for disparity detecting neurones in the human visual system. Joumal of Physiology, $1972,255,437-455$.

CoRnsweEt, T. N. The staircase-method in psychophysics. American Journal of Psychology, 1962, 75, 485-491.

Findlay, J. M., \& Parker, D. M. An investigation of visual orientation constancy using orientation-specific properties of acuity and adaptation. Perception, 1972, 1, 305-313.

Graham, C. H., Riggs, L. A., Mueller, C. G., \& Solomon, R. L. Precision of stereoscopic settings as influenced by distance of target from a fiducial line. Journal of Psychology, $1949,27,203-207$.

Graham, C. H. Vision and visual perception. New York: Wiley, 1965.

Hubet, D. H., \& Wiesel, T. N. Cells sensitive to binocular depth in area 18 of the macaque monkey cortex. Nature, 1970, 225, 41-42.

MiLleR. E. F. Counterrolling of the human eyes produced by head tilt with respect to gravity. Acta Otolanyngology, 1962 , 54, 479-501.

Mitchell, D. E., \& BAKer, A. G. Stereoscopic aftereffects: Evidence for disparity-specitic neurones in the human visual system. Vision Research, 1973. 13, 2273-2288.

Mitchell, D. E., \& Blakemore, C. B. The site of orientational constancy. Perception, 1972, 1, 315-320.
Mitchell. D. E., \& O'Hagan, S. Accuracy of stereoscopic localization of small line segments that differ in size or orientation for the two eyes. Vision Research, 1972, 12, 437-454.

Mueller. C. G., \& LloYd, V. V. Stereoscopic acuity for various levels of illumination. Proceedings of the National Academy of Sciences. 1948. 34, 223-227.

Nikara, T.. Bishop, P. O., \& Pettigrew, J. D. Analysis of retinal correspondence by studying receptive fields of binocular single units in the cat striate cortex. Experimental Brain Research, 1968, 6, 353-372.

OGLE, K. N. Spatial localization through binocular vision. In H. Davson (Ed.), The eye. New York: Academic Press, 1962. Pp. 271-324.

\section{NOTE}

1. This formulation does not take into account the lateral separation between the test rods. It can be shown from geometry that increases in lateral separation between rods of fixed length and orientation reduce the proportion of the contours of those rods over which horizontal disparity can be calculated. Because in our experiments this separation was only $10^{\prime}$, we have not included this factor in Equation 1.

(Received for publication December 22, 1975; accepted March 23, 1976.) 